Walter BOSSERT and Yves SPRUMONT

Centre de recherche et développement en économique

C.P. 6128 , succursale Centre-ville Montréal QC H3C 3J7 
CAHIER 01-2001

\title{
NON-DETERIORATING CHOICE*
}

\author{
Walter BOSSERT ${ }^{1}$ and Yves SPRUMONT $^{1}$
}

$1 \quad$ Centre de recherche et développement en économique (C.R.D.E.) and Département de sciences économiques, Université de Montréal

January 2001

* Financial support from the Social Sciences and Humanities Research Council of Canada and the Centre de recherche et développement en économique of the Université de Montréal is gratefully acknowledged. 


\title{
RÉSUMÉ
}

Une règle de sélection associe à tout ensemble d'options réalisables et tout statu quo y appartenant un sous-ensemble des options réalisables. Une telle règle est non-dégradante s'il existe un ordre sur l'ensemble de toutes les options concevables pour lequel les options sélectionnées ne sont jamais pires que le statu quo. Nous caractérisons de telles règles dans des contextes abstrait et économique.

Mots clés : fonctions de choix, rationalité individuelle, statu quo

\begin{abstract}
We analyze an alternative to the standard rationalizability requirement for observed choices by considering non-deteriorating selections. A selection function is a generalization of a choice function where selected alternatives may depend on a reference (or status quo) alternative in addition to the set of feasible options. A selection function is non-deteriorating if there exists an ordering over the universal set of alternatives such that the selected alternatives are at least as good as the reference option. We characterize non-deteriorating selection functions in an abstract framework and in an economic environment.
\end{abstract}

Key words : choice functions, individual rationality, status quo 


\section{Introduction}

In traditional choice theory, the decision maker is assumed to possess a preference relation over all conceivable alternatives. It then chooses from any feasible set it may face the maximal elements of that relation in that set. The testable restrictions implied by this preference maximization hypothesis are well known: they constitute the so-called theory of revealed preference pioneered by Samuelson (1938), Houthakker (1950), Arrow (1959), Richter (1966), and Sen (1971), among others. Normatively appealing as it may be, preference maximization does not have strong empirical support; in fact, rather systematic violations have been recorded in a variety of contexts (see, for example, the surveys by Camerer, 1994, and Shafir and Tversky, 1995). Therefore, it is useful to analyze alternative models of choice.

In this paper, we explore one such alternative. We deviate from standard choice theory by assuming that, in each feasible set, there exists a reference alternative which, in addition to the feasible set itself, may influence the choice of the agent. The reference alternative can be interpreted in various ways, depending on the structure of the problem. In general, we can think of it as an option representing the status quo. This interpretation is appropriate, for example, in dynamic choice situations where consecutive choices are observed. In a multi-stage choice problem of that nature, a plausible reference alternative at a given stage is the one that has been selected in the previous stage, provided it remains feasible. In economic environments (such as economies with private goods), it is natural to consider endowment vectors to be the reference alternatives relevant to the choice.

The idea that the status quo matters is, of course, not novel. It dates back at least to Simon $(1955,1956)$ and remained an important theme in the literature on bounded rationality ever since. Only recently, however, did Zhou (1997) approach it from the revealed preference angle. Zhou asked the following general question: what testable restrictions, if any, are implied by theories of bounded rationality where the status quo plays a role? He then focused on one such theory according to which the agent maximizes a "quasicomplete" transitive relation over the set of alternatives that are better than the status quo.

The alternative rationality requirement we consider in this paper merely demands that there exist an ordering (or indeed just a transitive preference relation) according to which the observed choices never make the agent worse off than the reference alternative. This requirement, which is devoid of any idea of maximization, is often referred to as individual rationality. However, to avoid confusion with the rationality requirement imposed 
in revealed preference theory, we prefer to speak of non-deteriorating choice. Though non-deteriorating choice is a weak behavioral assumption, it is far from being a vacuous requirement: as shown in this paper, it yields substantial testable restrictions on observed choices.

Our general model is basically the same as the one in Zhou (1997). A selection function assigns to each pair of a feasible set and a reference alternative (required to be an element of the feasible set) a set of chosen alternatives which is a subset of the feasible set. This selection function is non-deteriorating if there exists a preference ordering over the universal set of alternatives such that, for all elements in the domain of the selection function, all chosen alternatives are at least as good as the reference alternative. As is the case for rational choice, the formulation of necessary and sufficient conditions for non-deteriorating choice depends crucially on the domain of the selection function. We analyze two types of domains in this paper. The first deals with the case where no restrictions are imposed on the structure of the domain. Second, we discuss a more structured environment where alternatives are represented as vectors in a Euclidean space. These vectors can be interpreted as consumption bundles and, therefore, it is natural to impose additional regularity requirements such as continuity, monotonicity, or convexity on preference relations for which the observed choices are non-deteriorating.

In Section 2, we present the abstract version of our model. We provide a necessary and sufficient condition that is analogous to Richter's (1966) congruence axiom characterizing rational choice. In order to avoid degenerate situations where any selection function is declared non-deteriorating by employing the universal indifference relation, we require the relation for which the selection function is non-deteriorating to be antisymmetric in that section.

Section 3 deals with economic environments. This more structured version of our model lends itself to empirical testing. With this application in mind, we restrict the domain of the selection function to be finite, in which case an equivalent formulation in terms of data sets can be employed. Again, we provide necessary and sufficient conditions for non-deteriorating choice, where natural restrictions are imposed on the relation for which the selections are non-deteriorating. While we do not assume antisymmetry in this section, we employ monotonicity and convexity assumptions and characterize nondeteriorating choice in three cases: for strictly monotonic preferences, for strictly convex preferences, and for preference orderings that are strictly monotonic and strictly convex. It should be noted that adding continuity to the list of properties of the relation does not have any consequences: continuity by itself imposes no testable restrictions at all (again, 
the universal indifference relation can be employed to demonstrate this observation) and, in the context of finite economic domains, it is redundant when strict monotonicity or strict convexity are imposed in the first place.

Section 4 concludes, and the equivalence between selection functions and data sets in the case of economic environments with finite domains is established in the Appendix.

\section{Abstract Choices}

Let $\mathbb{N}\left(\mathbb{N}_{0}\right)$ denote the set of positive (nonnegative) integers and, for $m \in \mathbb{N}$, let $\mathbb{R}^{m}$ denote Euclidean $m$-space. Let $X$ be a nonempty set of alternatives. The set of all nonempty subsets of $X$ is denoted by $\mathcal{P}(X)$. A quasi-ordering over $X$ is a reflexive and transitive binary relation $R \subseteq X \times X$, and an ordering is a complete quasi-ordering. The symmetric and asymmetric factors of $R$ are denoted by $I$ and $P$. The relation $R$ is antisymmetric if, for all $x, y \in X, x I y$ implies $x=y$.

A (selection) problem is a pair $(S, x)$ such that $S \in \mathcal{P}(X)$ and $x \in S$. We interpret $S$ as the feasible set of alternatives and $x$ as the reference point for the problem under consideration. The domain of problems is denoted by $\mathcal{D}$. We assume that $\mathcal{D}$ is nonempty but otherwise, no restrictions are imposed in this section.

A selection function is a mapping $C: \mathcal{D} \rightarrow \mathcal{P}(X)$ such that $C(S, x) \subseteq S$ for all $(S, x) \in$ $\mathcal{D}$. Note that $C(S, x)$ could contain several alternatives but single-valued choices are included as a special case.

Given an ordering $R$ over $X$, a selection function $C$ is non-deteriorating for $R$ if $x R y$ for all $(S, y) \in \mathcal{D}$ and for all $x \in C(S, y)$.

In the characterization result of this section, we restrict attention to selection functions that are non-deteriorating for an antisymmetric ordering to prevent the concept of nondeterioration from becoming vacuous. Clearly, without a restriction such as antisymmetry, any selection function would be non-deteriorating for the universal indifference relation.

An obvious necessary condition for a selection function to be non-deteriorating for an antisymmetric ordering is that if, for some problem, an alternative $y$ is chosen when $x \neq y$ is the reference alternative, then $x$ cannot be chosen for any problem where $y$ is the reference alternative. Consider the following example.

Example 1. Let $X=\{x, y\}$ and $\mathcal{D}=\{(\{x, y\}, x),(\{x, y\}, y)\}$, and define the selection function $C$ by letting $C(\{x, y\}, x)=\{y\}$ and $C(\{x, y\}, y)=\{x\}$. It is immediate that $C$ cannot be non-deteriorating for any antisymmetric relation $R$ over $X$. 
On the other hand, the above-described requirement is not sufficient for non-deteriorating choice for a transitive and antisymmetric relation, as demonstrated in the following example.

Example 2. Let $X=\{x, y, z\}$ and $\mathcal{D}=\{(S, w): S \in \mathcal{P}(X),|S|=2$, and $w \in S\}$. Define the selection function $C$ by letting

$$
\begin{aligned}
& C(\{x, y\}, x)=C(\{x, y\}, y)=\{x\}, \\
& C(\{y, z\}, y)=C(\{y, z\}, z)=\{y\}, \\
& C(\{x, z\}, x)=C(\{x, z\}, z)=\{z\} .
\end{aligned}
$$

$C$ satisfies the above condition but it is not non-deteriorating for any transitive and antisymmteric relation $R$.

Example 2 suggests that no cycles of revealed preference should occur. Therefore, a necessary and sufficient condition has to take into consideration the transitive closure of direct revealed preferences. To formulate such a necessary and sufficient condition, we introduce the following direct and indirect revealed preference relations associated with a selection function $C$. These relations are analogous to those that are familiar from the literature on rational choice.

The direct revealed preference relation $R_{C}$ associated with $C$ is defined as follows. For all $x, y \in X, x R_{C} y$ if there exists $S \in \mathcal{P}(X)$ such that $(S, y) \in \mathcal{D}$ and $x \in C(S, y)$.

The (indirect) revealed preference relation $\bar{R}_{C}$ corresponding to $C$ is the transitive closure of $R_{C}$. Formally, it is defined by letting, for all $x, y \in X, x \bar{R}_{C} y$ if there exist $K \in \mathbb{N}$ and $x^{0}, \ldots, x^{K} \in X$ such that $x=x^{0}, y=x^{K}$, and $x^{k-1} R_{C} x^{k}$ for all $k \in\{1, \ldots, K\}$. Clearly,

$$
R_{C} \subseteq \bar{R}_{C} .
$$

The following axiom is analogous to Richter's (1966) congruence axiom which is necessary and sufficient for the rationalizability of a choice function with an arbitrary domain. ND-Congruence. For all $x, y \in X$ such that $x \neq y$, for all $S \in \mathcal{P}(X)$ such that $(S, x) \in \mathcal{D}$, if $x \bar{R}_{C} y$, then $y \notin C(S, x)$.

Before stating the main theorem of this section, we provide the following lemma which will be used in the proof.

Lemma 1. If $R$ is an antisymmetric ordering for which $C$ is a non-deteriorating selection function, then $\bar{R}_{C} \subseteq R$.

Proof. Suppose $C$ is non-deteriorating for an antisymmetric ordering $R$. 
First, we show that $R_{C} \subseteq R$. Suppose $x R_{C} y$ for $x, y \in X$. Then there exists $S \in \mathcal{P}(X)$ such that $(S, y) \in \mathcal{D}$ and $x \in C(S, y)$. Because $C$ is non-deteriorating for $R$, this implies $x R y$.

Now suppose $x \bar{R}_{C} y$ for $x, y \in X$. Then there exist $K \in \mathbb{N}$ and $x^{0}, \ldots, x^{K} \in X$ such that $x=x^{0}, y=x^{K}$, and $x^{k-1} R_{C} x^{k}$ for all $k \in\{1, \ldots, K\}$ Because $R_{C} \subseteq R$, it follows that $x^{k-1} R x^{k}$ for all $k \in\{1, \ldots, K\}$. Because $R$ is transitive, this implies $x R y$.

The above lemma is analogous to the relationship between the revealed preference relation and a rationalizing relation in the context of rational choice (see, for example, Samuelson, 1938, 1948, and Richter, 1971).

We are now ready to prove:

Theorem 1. There exists an antisymmetric ordering $R$ for which $C$ is non-deteriorating if and only if $C$ satisfies $N D$-Congruence.

Proof. (a) "Only if." Suppose $C$ is non-deteriorating for an antisymmetric ordering $R$ over $X$. By way of contradiction, suppose $C$ violates ND-Congruence. Then there exist $x, y \in X$ with $x \neq y$ and $S \in \mathcal{P}(X)$ such that $(S, x) \in \mathcal{D}, x \bar{R}_{C} y$, and $y \in C(S, x)$. By definition, this implies $y R_{C} x$ and, by (1) and Lemma 1, $y R x$. Because $x \bar{R}_{C} y$, Lemma 1 implies $x R y$. Because $x \neq y$, this contradicts the antisymmetry of $R$.

(b) "If." Suppose $C$ satisfies ND-Congruence. First, we prove that $\bar{R}_{C}$ is antisymmetric. By way of contradiction, suppose there exist $x, y \in X$ with $x \neq y$ such that $x \bar{R}_{C} y$ and $y \bar{R}_{C} x$. Then there exist $K, L \in \mathbb{N}, x^{0}, \ldots, x^{K} \in X$, and $z^{0}, \ldots, z^{L} \in X$ such that $x=x^{0}=z^{L}, y=x^{K}=z^{0}, x^{k-1} R_{C} x^{k}$ for all $k \in\{1, \ldots, K\}$, and $z^{\ell-1} R_{C} z^{\ell}$ for all $\ell \in\{1, \ldots, L\}$. This implies $x \bar{R}_{C} z^{L-1}$. Because $z^{L-1} R_{C} z^{L}=x$, there exists $S \in \mathcal{P}(X)$ such that $(S, x) \in \mathcal{D}$ and $z^{L-1} \in C(S, x)$. But this contradicts ND-Congruence.

Now define the ordering $R^{*}$ over $X$ by letting, for all $x, y \in X, x R^{*} y$ if and only if $x \bar{R}_{C} y$ or $x=y$. Clearly, $R^{*}$ is reflexive and, because $\bar{R}_{C}$ is transitive and antisymmetric, so is $R^{*}$. By Szpilrajn's (1930) result on extending transitive and antisymmetric quasiorderings, there exists an antisymmetric ordering $R$ over $X$ such that

$$
R^{*} \subseteq R
$$

It remains to be shown that $C$ is non-deteriorating for $R$. Suppose $x \in C(S, y)$ for some $x, y \in X$ and $S \in \mathcal{P}(X)$ such that $(S, y) \in \mathcal{D}$. Then $x R_{C} y$ by definition of the direct revealed preference relation. Furthermore, by (1), Lemma 1, and (2), we have

$$
R_{C} \subseteq \bar{R}_{C} \subseteq R^{*} \subseteq R
$$


and, hence, $x R y$.

As is apparent from the proof of Theorem 1, ND-Congruence remains necessary and sufficient for non-deteriorating choice if the assumption that $R$ is an ordering is weakened to transitivity. Note that $\bar{R}_{C}$ is a transitive relation for which a selection function satisfying ND-Congruence is non-deteriorating, and the part of the above proof extending $\bar{R}_{C}$ to the ordering $R$ could be omitted if $R$ is merely required to be transitive. Furthermore, the "only if" part of the proof and Lemma 1 remain valid if $R$ is transitive without having any further properties. Thus, the testable restrictions identified in the theorem are robust with respect to weakening the requirements on the relation $R$.

\section{Economic Choices}

In this section, we turn to economic environments: the universal set $X$ is $\mathbb{R}^{m}, m$ dimensional vectors are interpreted as commodity bundles, and preferences are orderings over $\mathbb{R}^{m}$. Our notation for vector inequalities is $\geq,>, \gg$.

The following concept will prove convenient. A data set is a mapping $(s, e, c)$ from some nonempty and finite index set $T$ into $\mathcal{P}\left(\mathbb{R}^{m}\right) \times \mathbb{R}^{m} \times \mathbb{R}^{m}$ such that, for each $t \in T, e(t) \in s(t)$ and $c(t) \in s(t)$. We interpret $s(t)$ as the feasible set at "time" $t$ or at "observation" $t$, and $e(t)$ and $c(t)$ as the endowment and consumption choice at $t$. This formulation is in fact equivalent to the selection function formulation used in Section 2, provided that the domain under consideration is finite; see the Appendix for details. From now on, we fix $T$.

If $(s, e, c)$ is a data set and $R$ is a preference ordering on $\mathbb{R}^{m}$, we say that $(s, e, c)$ is non-deteriorating for $R$ if, for all $t \in T, c(t) R e(t)$.

Our goal is to identify necessary and sufficient conditions for a data set to be nondeteriorating for some preference ordering $R$ satisfying suitable restrictions. We say that $R$ is strictly monotonic if, for all $x, y \in \mathbb{R}^{m}, x>y \Rightarrow x P y$. We call $R$ strictly convex if, for all distinct $x, y \in \mathbb{R}^{m}$ and for all $\lambda \in(0,1), x R y \Rightarrow[\lambda x+(1-\lambda) y] P y$. We shall consider strictly monotonic orderings in the first subsection and strictly convex orderings in the second. The third subsection deals with orderings that are strictly monotonic and strictly convex. 


\subsection{Strict Monotonicity}

For any $Y \subseteq \mathbb{R}^{m}$, define up $Y=\left\{x \in \mathbb{R}^{m}\right.$ : there exists $y \in Y$ such that $\left.x \geq y\right\}$ and low $Y=\{y \in Y$ : there is no $x \in Y$ such that $x<y\}$. Consider the following condition on a data set $(s, e, c)$.

No Strong Dominance. For every nonempty $Q \subseteq T$ such that $c(Q)=\operatorname{low} c(Q)$ and $e(Q) \subseteq$ up $c(Q)$, there is a nonempty $Q^{0} \subseteq Q$ such that $e\left(Q^{0}\right) \subseteq c\left(Q^{0}\right)$.

If we restrict attention to those subsets of $T$ which are singletons, this condition implies that for all $t \in T, e(t) \geq c(t) \Rightarrow e(t)=c(t)$, a condition we call No Direct Dominance. This condition is necessary if $(s, e, c)$ is to be non-deteriorating for a strictly monotonic preference ordering. No Strong Dominance, however, is more demanding, as the following example shows.

Example 3. Let $m=2, T=\{1,2\}$, and $s(1)=s(2)=\mathbb{R}^{2}$. Assume $e(1)=c(2)=(2,0)$, $c(1)=(0,1)$, and $e(2)=(1,2)$. It is easily seen that there is no strictly monotonic preference ordering for which this data set is non-deteriorating. No Direct Dominance is satisfied but No Strong Dominance is violated.

On the other hand, No Strong Dominance does not rule out all forms of dominance of the endowments over the choices, as our next example illustrates.

Example 4. Let again $m=2, T=\{1,2\}$, and $s(1)=s(2)=\mathbb{R}^{2}$. Assume $e(1)=(1,0)$, $c(1)=(0,1)$, and $e(2)=c(2)=(2,2)$. Note that for each $t$ there is a $t^{\prime}$ for which $e\left(t^{\prime}\right) \geq c(t)$, and this inequality is strict for some pair of indices, namely $t=1, t^{\prime}=2$. Yet, it is easy to find a strictly monotonic preference ordering for which this data set is non-deteriorating. No Strong Dominance is satisfied.

We are now ready to prove:

Theorem 2. There exists a strictly monotonic preference ordering $R$ for which $(s, e, c)$ is non-deteriorating if and only if $(s, e, c)$ satisfies No Strong Dominance.

Proof. Since the feasible set mapping $s$ plays no role in the proof, we drop it from our notation.

(a) "Only if." Fix a data set $(e, c)$ and let $R$ be a strictly monotonic preference ordering for which $(e, c)$ is non-deteriorating, that is,

$$
\text { for all } t \in T, c(t) R e(t) \text {. }
$$

Let $Q \subseteq T$ be a nonempty set such that

$$
c(Q)=\operatorname{low} c(Q)
$$


and

$$
e(Q) \subseteq u p c(Q)
$$

Let $f(Q)=e(Q) \cup c(Q)$ and define $W$ to be the set of worst elements in $f(Q)$ according to $R$, that is, $W=\{x \in f(Q)$ : for all $y \in f(Q), y R x\}$. Since $R$ is an ordering and $f(Q)$ is nonempty and finite, $W$ is nonempty. By strict monotonicity of $R, W \subseteq$ low $f(Q)$. By (4) and (5), low $f(Q)=$ low $c(Q)=c(Q)$. Therefore, $W \subseteq c(Q)$. Define the nonempty set

$$
Q^{0}=\{t \in Q: c(t) \in W\}
$$

Since $W \subseteq c(Q), W=c\left(Q^{0}\right)$. To complete the proof, it suffices to check that $e\left(Q^{0}\right) \subseteq$ $c\left(Q^{0}\right)$. Fix $t \in Q^{0}$. By definition, $c(t) \in W$, and it follows from $(3)$ that $e(t) \in W=c\left(Q^{0}\right)$, as desired.

(b) "If." Let $(e, c)$ satisfy No Strong Dominance.

Step 1. We first claim that No Strong Dominance implies the following condition. For every nonempty $Q \subseteq T$ such that $c(Q)=$ low $c(Q)$ and $e(Q) \subseteq$ up $c(Q)$, there is a nonempty set $\bar{Q}^{0} \subseteq Q$ such that $e\left(\bar{Q}^{0}\right) \subseteq c\left(\bar{Q}^{0}\right)$ and

$$
c\left(Q \backslash \bar{Q}^{0}\right) \cap c\left(\bar{Q}^{0}\right)=\emptyset .
$$

To prove this claim, let $Q \subseteq T$ be a nonempty set such that $c(Q)=$ low $c(Q)$ and $e(Q) \subseteq u p c(Q)$. By No Strong Dominance, there is a nonempty $Q^{0} \subseteq Q$ such that

$$
e\left(Q^{0}\right) \subseteq c\left(Q^{0}\right)
$$

Let $Q^{0 *}$ be the union of all nonempty subsets $Q^{0}$ of $Q$ satisfying property (7). Because that property is preserved under unions, $Q^{0 *}$ possesses it. If $c\left(Q \backslash Q^{0 *}\right) \cap c\left(Q^{0 *}\right)=\emptyset$, we are done. Otherwise, let

$$
\bar{Q}=Q \backslash\left\{q \in Q^{0 *}: \exists t \in Q \backslash Q^{0 *} \text { such that } c(q)=c(t)\right\}
$$

Clearly, $\emptyset \neq \bar{Q} \subseteq Q$ and $c(\bar{Q})=c(Q)$. Hence, $c(\bar{Q})=$ low $c(\bar{Q})$ and $e(\bar{Q}) \subseteq e(Q) \subseteq u p$ $c(Q)=u p c(\bar{Q})$. We may therefore apply No Strong Dominance again and conclude that there exists a nonempty $\bar{Q}^{0} \subseteq \bar{Q}$ such that $e\left(\bar{Q}^{0}\right) \subseteq c\left(\bar{Q}^{0}\right)$. To complete the proof, we check property (6). By definition of $Q^{0 *}$, we know that $\bar{Q}^{0} \subseteq Q^{0 *}$. Since $\bar{Q}^{0} \subseteq \bar{Q} \cap Q^{0 *}$, it follows from the definition of $\bar{Q}$ that $c\left(Q \backslash \bar{Q}^{0}\right) \cap c\left(\bar{Q}^{0}\right)=\emptyset$, as desired.

Step 2. Let $f(T)=e(T) \cup c(T)$. We define an algorithm that yields a partition $\left\{X_{0}, X_{1}, \ldots, X_{J}\right\}$ of $f(T)$ and construct a strictly monotonic preference ordering $R$ whose indifference sets in $f(T)$ are, from worst to best, $X_{0}, X_{1}, \ldots, X_{J}$. 
To define the algorithm, let $F_{0}=f(T)$. At step $i \in \mathbb{N}_{0}$, we construct $F_{i+1}$ by removing from $F_{i}$ a set $X_{i}$ that we define as follows. First, let

$$
\begin{gathered}
X^{1}\left(F_{i}\right)=\left\{x \in \text { low } F_{i}: \text { there is no } t \in T \text { such that } x=c(t)\right\}, \\
X^{2}\left(F_{i}\right)=\left\{x \in \text { low } F_{i}: \text { for all } t \in T, x=c(t) \Rightarrow e(t) \notin F_{i}\right\} .
\end{gathered}
$$

If both of these sets are empty, define $X^{3}\left(F_{i}\right)$ as follows. If $F_{i}=\emptyset$, let $X^{3}\left(F_{i}\right)=\emptyset$. Otherwise, let

$$
T_{i}=\left\{t \in T: c(t) \in \text { low } F_{i}\right\} .
$$

Clearly, $c\left(T_{i}\right)=$ low $c\left(T_{i}\right)$. Moreover, since $X^{1}\left(F_{i}\right)=X^{2}\left(F_{i}\right)=\emptyset$, we know that $T_{i} \neq \emptyset$ and $e\left(T_{i}\right) \subseteq$ up $c\left(T_{i}\right)$. By No Strong Dominance and Step 1, there must exist a nonempty $T_{i}^{0} \subseteq T_{i}$ such that

$$
e\left(T_{i}^{0}\right) \subseteq c\left(T_{i}^{0}\right)
$$

and

$$
c\left(T_{i} \backslash T_{i}^{0}\right) \cap c\left(T_{i}^{0}\right)=\emptyset
$$

Pick any such set and let

$$
X^{3}\left(F_{i}\right)=c\left(T_{i}^{0}\right)
$$

Define, for each $i \in \mathbb{N}_{0}$,

$$
X_{i}= \begin{cases}X^{1}\left(F_{i}\right) & \text { if } X^{1}\left(F_{i}\right) \neq \emptyset \\ X^{2}\left(F_{i}\right) & \text { if } X^{1}\left(F_{i}\right)=\emptyset \neq X^{2}\left(F_{i}\right) \\ X^{3}\left(F_{i}\right) & \text { if } X^{1}\left(F_{i}\right)=X^{2}\left(F_{i}\right)=\emptyset .\end{cases}
$$

First, note that there is a nonnegative integer $J<|f(T)|$ such that $X_{i} \neq \emptyset=X_{j}$ whenever $i \leq J<j$. Moreover, each $x$ in $f(T)$ belongs to $X_{i}$ for one and only one $i \in\{0, \ldots, J\}$.

Second, observe that for each $i \in\{0, \ldots, J\}$, we have $X_{i} \subseteq$ low $F_{i}$. This implies that, for all $x, y \in X_{i}$, neither $x<y$ nor $x>y$.

These two observations allow us to construct a strictly monotonic preference ordering $R$ on $\mathbb{R}^{m}$ whose indifference sets in $f(T)$ are, from worst to best, $X_{0}, \ldots, X_{J}$.

Step 3. We show that $(e, c)$ is non-deteriorating for $R$. To that end, we claim that, for all $i \in\{0, \ldots, J\}$, and for all $t \in T$,

$$
c(t) \in X_{i} \Rightarrow \text { there exists } j \in\{0, \ldots, i\} \text { such that } e(t) \in X_{j} .
$$

To check this claim, fix $t \in T$ and assume $c(t) \in X_{i}$. By (8) and (12), $X^{1}\left(F_{i}\right)=\emptyset$. If $X_{i}=X^{2}\left(F_{i}\right),(8),(9)$, and (12) imply that $e(t) \notin F_{i}$. Hence, $e(t) \in X_{j}$ for some $j<i$, and 
we are done. Finally, assume $X_{i}=X^{3}\left(F_{i}\right)$. Since this set is nonempty (as $c(t)$ belongs to it), there is a nonempty set $T_{i}^{0}$ satisfying (10) and (11) such that $X_{i}=c\left(T_{i}^{0}\right)$. By (11), $c(t) \in c\left(T_{i}^{0}\right) \Rightarrow t \in T_{i}^{0}$. Hence, from $(10), e(t) \in c\left(T_{i}^{0}\right)$ and, therefore, $e(t) \in X_{i}$.

\subsection{Strict Convexity}

If $Y$ is a convex subset of $\mathbb{R}^{m}$, an extreme point of $Y$ is a point $y \in Y$ such that

$$
[x, z \in Y, \lambda \in(0,1) \text {, and } y=\lambda x+(1-\lambda) z] \Rightarrow[x=y=z]
$$

For any $Y \subseteq \mathbb{R}^{m}$, we let co $Y$ denote the convex hull of $Y$ and we denote by extco $Y$ the set of extreme points of $c o Y$. Consider the following condition on a data set $(s, e, c)$.

No Strong Inclusion. For every nonempty $Q \subseteq T$ such that $c(Q)=\operatorname{extco} c(Q)$ and $e(Q) \subseteq \operatorname{coc}(Q)$, there is a nonempty $Q^{0} \subseteq Q$ such that $e\left(Q^{0}\right) \subseteq c\left(Q^{0}\right)$.

The formal similarity with No Strong Dominance is worth pointing out: the "extco" operator replaces "low" while "co" replaces "up." In the example below, No Strong Inclusion is violated.

Example 5. In all examples, $m=2$ and $s(t)=\mathbb{R}^{2}$ for all $t \in T$.

(a) Let $T=\{1,2,3\}$. Suppose $c(1)=(0,0), c(2)=(4,0), c(3)=(0,4)$, while $e(1)=$ $(1,1), e(2)=(2,1), e(3)=(1,2)$. Since $e(T)$ is in the interior of the convex hull of $c(T)$, No Strong Inclusion is clearly violated. There is no strictly convex preference ordering for which this data set is non-deteriorating. For suppose $R$ were such an ordering. Assume, without loss of generality, that $c(t) R c(1)$ for all $t \in T$. By strict convexity, $e(1) P c(1)$, a contradiction.

(b) Again, let $T=\{1,2,3\}$. Suppose $c(1)=e(2)=(0,0), c(2)=e(3)=(4,0)$, $c(3)=(0,4)$, and $e(1)=(1,1)$. It is easily checked that there is no strictly convex preference ordering for which this data set is non-deteriorating. No Strong Inclusion is violated.

(c) Finally, let $T=\{1,2,3,4\}$. Define $c$ and $e$ on $\{1,2,3\}$ as in (b) and let $c(4)=(0,0)$, $e(4)=(0,4)$. Here again, $c(T)=\operatorname{extco} c(T), e(T) \subseteq \cos c(T)$, but there is a nonempty set $T^{0} \subseteq T$ such that $e\left(T^{0}\right) \subseteq c\left(T^{0}\right)$, namely, $T^{0}=\{2,3,4\}$. No Strong Inclusion is nevertheless violated. Contrary to the previous examples, "the violation occurs in a strict subset of $T$," namely, $Q=\{1,2,3\}$ : there is no nonempty $Q^{0} \subseteq Q$ such that $e\left(Q^{0}\right) \subseteq c\left(Q^{0}\right)$. There is no strictly convex preference ordering for which this data set is non-deteriorating. 
No Strong Inclusion does not rule out all instances of inclusion of the endowments in the convex hull of the choices. The following example makes this point clear.

Example 6. Let $m=2$ and $s(t)=\mathbb{R}^{2}$ for all $t \in T$.

(a) Let $T=\{1,2,3\}$ and $c(1)=e(2)=(0,0), c(2)=e(3)=(4,0), c(3)=e(1)=(0,4)$. Obviously, there is a strictly convex preference ordering for which this data set is nondeteriorating: it suffices to declare all endowment and choices in the data set indifferent. No Strong Inclusion is satisfied.

(b) Let $T=\{1,2,3\}$ and $c(1)=(0,0), c(2)=e(3)=(4,0), c(3)=e(2)=(0,4)$, and $e(1)=(1,1)$. This example is quite close to Example 5 (b) above. Here, however, our condition is satisfied and there is a strictly convex preference ordering for which the data set is non-deteriorating. We are now ready to state:

Theorem 3. There exists a strictly convex preference ordering $R$ for which $(s, e, c)$ is non-deteriorating if and only if $(s, e, c)$ satisfies No Strong Inclusion.

Proof. Mutatis mutandis, the proof is the same as that of Theorem 2. The only required changes are as follows: i) replace every instance of "low" with "extco" and every instance of "up" with "co;" ii) replace every instance of "monotonic(ity)" with "convex(ity)" and every instance of "No Strong Dominance" with "No Strong Inclusion;" and iii) in the second observation in Step 2, replace the phrase "for all $x, y \in X_{i}$, neither $x<y$ nor $x>y$ " with "every $x \in X_{i}$ is an extreme point of the convex hull of $X_{i}$."

\subsection{Strict Monotonicity and Strict Convexity}

If we require a preference ordering to be strictly monotonic and strictly convex, the conjunction of No Strong Dominance and No Strong Inclusion is not sufficient to guarantee non-deteriorating choice. Consider the following example.

Example 7. Let $m=2, T=\{1,2\}, s(t)=\mathbb{R}^{2}$ for all $t \in T, c(1)=(4,0), c(2)=(0,4)$, $e(1)=(3,2)$, and $e(2)=(2,3)$. This data set satisfies No Strong Dominance and No Strong Inclusion but it is not non-deteriorating for any strictly monotonic and strictly convex preference ordering. For suppose $R$ were such an ordering. Assume, without loss of generality, that $c(2) R c(1)$. By strict monotonicity and strict convexity, $e(1)=$ $(3,2) P(2,2)=\left[\frac{1}{2} c(1)+\frac{1}{2} c(2)\right] P c(1)$, a contradiction.

The following axiom turns out to be necessary and sufficient for non-deteriorating choice, given the properties of preferences imposed in this subsection. 
No Extended Dominance. For every nonempty $Q \subseteq T$ such that $c(Q)=$ extco low co $c(Q)$ and $e(Q) \subseteq$ up co $c(Q)$, there is a nonempty $Q^{0} \subseteq Q$ such that $e\left(Q^{0}\right) \subseteq c\left(Q^{0}\right)$.

Before stating the characterization result of this subsection formally, we present the following lemma. It introduces a property of the operator "extco low co" that will be used in the proof.

Lemma 2. For all nonempty $Y \subseteq \mathbb{R}^{m}$, extco low co $Y \subseteq Y$.

Proof. Suppose

$$
x \in \text { extco low co } Y \text {. }
$$

This implies $x \in$ co $Y$. Hence, there exist a positive integer $K, K$ distinct points $x^{1}, \ldots, x^{K} \in Y$, and positive numbers $\alpha^{1}, \ldots, \alpha^{K}$ such that $\sum_{k=1}^{K} \alpha^{k}=1$ and $x=$ $\sum_{k=1}^{K} \alpha^{k} x^{k}$

If $K=1$, then $x=x^{1} \in Y$ and we are done. Now suppose $K>1$ and, by way of contradiction, $x \notin Y$. By (13), there exists $k \in\{1, \ldots, K\}$ such that $x^{k} \notin$ low co $Y$, say, $k=1$. Because $x^{1} \in Y \subseteq c o Y$, this means that there exists $y^{1} \in$ co $Y$ such that $y^{1}<x^{1}$. Define $y=\alpha^{1} y^{1}+\sum_{k=2}^{K} \alpha^{k} x^{k}$. Clearly $y \in \operatorname{co} Y$ and $y<x$. Therefore $x \notin$ low co $Y$, contradicting (13) since obviously extco low co $Y \subseteq$ low co $Y$.

The following theorem characterizes non-deteriorating choice for strictly monotonic and strictly convex preferences. Though the structure of the proof is analogous to that of Theorem 2, we present it in detail because the complexity of the operator "extco low co" necessitates some more elaborate arguments than those employed in the proof of Theorem 2 .

Theorem 4. There exists a strictly monotonic and strictly convex preference ordering $R$ for which $(s, e, c)$ is non-deteriorating if and only if $(s, e, c)$ satisfies No Extended Dominance.

Proof. Again, because the mapping $s$ is irrelevant for the proof, we omit it from our notation.

(a) "Only if." Fix a data set $(e, c)$, and let $R$ be a strictly monotonic and strictly convex ordering for which $(e, c)$ is non-deteriorating. Therefore,

$$
\text { for all } t \in T, c(t) R e(t) \text {. }
$$

Let $Q \subseteq T$ be a nonempty set such that

$$
c(Q)=\text { extco low co } c(Q)
$$


and

$$
e(Q) \subseteq u p \operatorname{coc}(Q)
$$

Let $f(Q)=e(Q) \cup c(Q)$.

Step 1. We prove that

$$
\text { extco low co } f(Q) \subseteq \text { extco low co } c(Q) \text {. }
$$

Suppose

$$
x \in \text { extco low co } f(Q) \text {. }
$$

By Lemma $2, x \in f(Q)$.

Because of (15), we need only show that $x \in c(Q)$.

Suppose, by contradiction, that $x \notin c(Q$ so that, necessarily, $x \in e(Q)$. We first claim that

$$
x \in \text { low co } c(Q) \text {. }
$$

Indeed, by (16), $x \in$ up co $c(Q)$. If $x \in$ up $\cos c(Q) \backslash \operatorname{coc}(Q)$, there exists $y \in \cos (Q) \subseteq c o$ $f(Q)$ such that $y<x$. Therefore $x \notin$ low co $f(Q)$, contradicting (18). Hence, $x \in$ co $c(Q)$. Now, if (19) is false, there exists $y \in c o c(Q) \subseteq \operatorname{co} f(Q)$ such that $y<x$, hence $x \notin$ low co $f(Q)$, contradicting (18).

Next, because of (19) and since $x \notin c(Q)=$ extco low co $c(Q)$, there exist an integer $K>1, K$ distinct points $x^{1}, \ldots, x^{K} \in$ low co $c(Q)$, and positive numbers $\alpha^{1}, \ldots, \alpha^{K}$ such that $\sum_{k=1}^{K} \alpha^{k}=1$ and $x=\sum_{k=1}^{K} \alpha^{k} x^{k}$. By (18), there exists $k \in\{1, \ldots, K\}$ such that $x^{k} \notin$ low co $f(Q)$, say, $k=1$. Because $x^{1} \in \operatorname{coc} c(Q) \subseteq \operatorname{co} f(Q)$, this means that there exists $y^{1} \in \operatorname{co} f(Q)$ such that $y^{1}<x^{1}$. Then again, $y=\alpha^{1} y^{1}+\sum_{k=2}^{K} \alpha^{k} x^{k} \in \operatorname{co} f(Q)$ and $y<x$. Hence, $x \notin$ low co $f(Q)$, contradicting (18). This completes the proof of (17).

Step 2. Let $W=\{x \in f(Q)$ : for all $y \in f(Q), y R x\}$. Because $R$ is an ordering and $f(Q)$ is finite, $W \neq \emptyset$. We claim that

$$
W \subseteq \text { extco low co } f(Q) .
$$

Suppose $x \in W$. First, we prove that $x \in \operatorname{low} \operatorname{co} f(Q)$. If not, there exists $y \in \operatorname{co} f(Q)$ such that $y<x$. The strict monotonicity of $R$ implies $x P y$. If $y \in f(Q), x$ cannot be a worst element in $f(Q)$, contradicting the assumption $x \in W$. If $y \in \operatorname{co} f(Q) \backslash f(Q)$, there exist an integer $K>1, K$ distinct points $x^{1}, \ldots, x^{K} \in f(Q)$, and $K$ positive numbers $\alpha^{1}, \ldots, \alpha^{K} \in f(Q)$ such that $\sum_{K=1}^{K} \alpha^{k}=1$ and $y=\sum_{k=1}^{K} \alpha^{k} x^{k}$. Because $R$ is strictly convex, $y P x^{k}$ for some $k \in\{1, \ldots, K\}$. Because $R$ is transitive, it follows that $x P x^{k}$, again contradicting the assumption that $x$ is a worst element in $f(Q)$. 
To complete the proof of (20), suppose, by way of contradiction, that $x \notin$ extco low co $f(Q)$. Because $x \in$ low co $f(Q)$ as just proven, this implies that there exist an integer $K>1, K$ distinct points $x^{1}, \ldots, x^{K} \in f(Q)$, and $K$ positive numbers $\alpha^{1}, \ldots, \alpha^{K} \in$ low co $f(Q)$ such that $\sum_{K=1}^{K} \alpha^{k}=1$ and $x=\sum_{k=1}^{K} \alpha^{k} x^{k}$. Because these points $x^{k}$ can be chosen to be in $f(Q)$, the convexity of $R$ again yields a contradiction to the assumption $x \in W$. This proves $(20)$.

Step 3. By (17) and (15), extco low co $f(Q) \subseteq$ extco low co $c(Q)=c(Q)$ and, hence,

$$
W \subseteq c(Q) .
$$

Defining $Q^{0}=\{t \in Q: c(t) \in W\}$, it follows from (21) that $W=c\left(Q^{0}\right)$. To complete the proof of part (a), it remains to be shown that $e\left(Q^{0}\right) \subseteq c\left(Q^{0}\right)$. If $t \in Q^{0}$, then $c(t) \in W$ and, from (14) and the definition of $W, e(t) \in W$. Because $W=c\left(Q^{0}\right)$, we conclude that $e(t) \in c\left(Q^{0}\right)$, as desired.

(b) "If." Let $(e, c)$ satisfy No Extended Dominance.

Step 1. Our first claim is that No Extended Dominance implies the following condition. For every nonempty $Q \subseteq T$ such that $c(Q)=$ extco low co $c(Q)$ and $e(Q) \subseteq$ up co $c(Q)$, there is a nonempty set $\bar{Q}^{0} \subseteq Q$ such that $e\left(\bar{Q}^{0}\right) \subseteq c\left(\bar{Q}^{0}\right)$ and $c\left(Q \backslash \bar{Q}^{0}\right) \cap c\left(\bar{Q}^{0}\right)=\emptyset$. Because the proof of this claim can be obtained from Step 1 in the proof of Theorem 2 by a change in notation, we omit it here.

Step 2. Let $f(T)=e(T) \cup c(T)$. We define an algorithm that yields a partition $\left\{X_{0}, X_{1}, \ldots, X_{J}\right\}$ of $f(T)$ and construct a strictly monotonic and strictly convex preference ordering $R$ whose indifference sets in $f(T)$ are, from worst to best, $X_{0}, X_{1}, \ldots, X_{J}$.

To define the algorithm, let $F_{0}=f(T)$. At step $i \in \mathbb{N}_{0}$, we construct $F_{i+1}$ by removing from $F_{i}$ a set $X_{i}$ that we define as follows. First, let

$$
\begin{gathered}
X^{1}\left(F_{i}\right)=\left\{x \in \text { extco low co } F_{i}: \text { there is no } t \in T \text { such that } x=c(t)\right\}, \\
X^{2}\left(F_{i}\right)=\left\{x \in \text { extco low co } F_{i}: \text { for all } t \in T, x=c(t) \Rightarrow e(t) \notin F_{i}\right\} .
\end{gathered}
$$

If both of these sets are empty, define $X^{3}\left(F_{i}\right)$ as follows. If $F_{i}=\emptyset$, let $X^{3}\left(F_{i}\right)=\emptyset$. Otherwise, let

$$
T_{i}=\left\{t \in T: c(t) \in \text { extco low co } F_{i}\right\} .
$$

We claim

$$
c\left(T_{i}\right)=\text { extco low co } c\left(T_{i}\right) .
$$

To prove (22), note first that

$$
\text { extco low co } c\left(T_{i}\right) \subseteq c\left(T_{i}\right)
$$


follows from Lemma 2. Because $X^{1}\left(F_{i}\right)=\emptyset$, it follows that

$$
c\left(T_{i}\right)=\text { ext low co } F_{i}
$$

To complete the proof of (22), it remains to show that extco low co $F_{i} \subseteq$ ext low co $c\left(T_{i}\right)$.

First, we prove that extco low co $F_{i} \subseteq$ low co $c\left(T_{i}\right)$. Let $x \in \operatorname{extco}$ low co $F_{i}$. By (24), $x \in c\left(T_{i}\right) \subseteq \operatorname{co~} c\left(T_{i}\right)$. Suppose $x \notin$ low co $c\left(T_{i}\right)$. Then there exists $y \in \operatorname{co} c\left(T_{i}\right) \subseteq$ co $F_{i}$ such that $y<x$, implying $x \notin$ low co $F_{i}$ and hence $x \notin$ extco low co $F_{i}$, the desired contradiction.

Now suppose $x \in$ extco low co $F_{i}$ but $x \notin$ extco low co $c\left(T_{i}\right)$. Because $x \in$ low co $c\left(T_{i}\right)$, there exist an integer $K>1, K$ distinct points $x^{1}, \ldots, x^{K} \in$ extco low co $c\left(T_{i}\right)$, and positive numbers $\alpha^{1}, \ldots, \alpha^{K}$ such that $\sum_{k=1}^{K} \alpha^{k}=1$ and $x=\sum_{k=1}^{K} \alpha^{k} x^{k}$. By (23) and $(24), x^{1}, \ldots, x^{K} \in c\left(T_{i}\right)=$ extco low co $F_{i}$, which implies $x \notin$ extco low co $F_{i}$, a contradiction which completes the proof of $(22)$.

Next, we prove that

$$
e\left(T_{i}\right) \subseteq \text { up co } c\left(T_{i}\right)
$$

Because $X^{2}\left(F_{i}\right)=\emptyset, e\left(T_{i}\right) \subseteq F_{i}$. Because $X^{1}\left(F_{i}\right)=\emptyset,(24)$ is true. Therefore, the proof of (25) is completed by showing that $F_{i} \subseteq$ up co extco low co $F_{i}$ or, equivalently, $F_{i} \subseteq u p$ co low co $F_{i}$.

Let $x \in F_{i}$. This implies $x \in$ co $F_{i}$. If $x \in$ low co $F_{i}$, then $x \in$ up co low co $F_{i}$ because low co $F_{i} \subseteq$ up co low co $F_{i}$. If $x \in$ co $F_{i} \backslash$ low co $F_{i}$, there exists $y \in$ co $F_{i}$ such that $y<x$, and we can choose $y \in$ low co $F_{i} \subseteq$ co low co $F_{i}$. Hence $x \in$ up co low co $F_{i}$, which completes the proof of (25).

By (22), (25), No Extended Dominance, and Step 1, there is a nonempty $T_{i}^{0} \subseteq T_{i}$ such that $e\left(T_{i}^{0}\right) \subseteq c\left(T_{i}^{0}\right)$ and $c\left(T_{i} \backslash T_{i}^{0}\right) \cap c\left(T_{i}^{0}\right)=\emptyset$. Pick any such set and let

$$
X^{3}\left(F_{i}\right)=c\left(T_{i}^{0}\right)
$$

Define, for each $i \in \mathbb{N}_{0}$,

$$
X_{i}= \begin{cases}X^{1}\left(F_{i}\right) & \text { if } X^{1}\left(F_{i}\right) \neq \emptyset \\ X^{2}\left(F_{i}\right) & \text { if } X^{1}\left(F_{i}\right)=\emptyset \neq X^{2}\left(F_{i}\right) \\ X^{3}\left(F_{i}\right) & \text { if } X^{1}\left(F_{i}\right)=X^{2}\left(F_{i}\right)=\emptyset\end{cases}
$$

The first observation of Step 2 of the proof of Theorem 2 is true again and the second observation is true if " $X_{i} \subseteq$ low $F_{i}$ " is replaced with " $X_{i} \subseteq$ extco low co $F_{i}$." The rest of the proof is identical to the rest of the proof of Theorem 2 . 


\section{Concluding Remarks}

Non-deteriorating choice is a consistency requirement that deviates from the standard rational choice model by not requiring choices to be best or maximal elements - selected alternatives merely have to be at least as good as a given reference option. This paper provides an analysis of this condition, and we identify testable restrictions on observed choices that allow us to check whether these choices conform to the behavioural hypothesis on which non-deteriorating choice is based.

We did not explicitly include continuity as a property of a preference ordering for which a data set is non-deteriorating. The reason is that continuity does not add any restrictions, neither by itself nor in conjunction with any combination of the other properties considered here. Note that if continuity alone is imposed on $R$, any data set is non-deteriorating for the universal indifference relation $R$. Furthermore, the orderings constructed in the proofs of Theorems 2 and 3 can be chosen to be continuous and, therefore, it is not necessary to impose this property explicitly.

The necessary and sufficient conditions presented in Section 3 are formulated directly in terms of the observed data sets rather than employing a revealed preference approach analogous to the one used in Section 2. Given that the economic environment has considerable structure as compared to the abstract model, we believe those formulations are more transparent than conditions involving transitive closures of direct revealed preferences. However, revealed preference approaches could be examined as alternatives. For example, in the case of strictly monotonic preference orderings, consider the following construction. For all $x, y \in e(T) \cup c(T)$, let $x R_{C}^{M} y$ if $x \geq y$ or there exists $t \in T$ such that $x=c(t)$ and $y=e(t)$. The transitive closure of $R_{C}^{M}$ is denoted by $\bar{R}_{C}^{M}$. The following axiom is necessary and sufficient for non-deteriorating choice for strictly monotonic preferences; details are available on request.

NDM-Congruence. For all $x, y \in e(T) \cup c(T)$, if $x \bar{R}_{C}^{M} y$ and $y \geq x$, then $y=x$.

We restrict attention to single-agent choice situations in this paper. A natural suggestion for an extension is to examine non-deteriorating choice, possibly together with other restrictions such as efficiency, in a multi-agent setting. This could be accomplished by using a framework analogous to the one employed in Bossert and Sprumont (2000) where core rationalizability is examined in a two-agent exchange economy. 


\section{Appendix}

In this Appendix, we illustrate that, in the case of an economic environment, the formulation of our problem in terms of a finite data set is equivalent to the selection function formulation with a finite domain.

We first show how a selection correspondence can be derived from a given (finite) data set. Suppose $X=\mathbb{R}^{m}, T=\{1, \ldots,|T|\}$ with $|T| \in \mathbb{N}$, and $(s, e, c): T \rightarrow \mathcal{P}(X) \times X \times X=$ $\mathcal{P}\left(\mathbb{R}^{m}\right) \times \mathbb{R}^{m} \times \mathbb{R}^{m}$ describe a data set. Define $\mathcal{D}=\{(S, x): \exists t \in T$ such that $(s(t), e(t))=$ $(S, x)\}$ and $C: \mathcal{D} \rightarrow \mathbb{R}^{m}$ by $C(S, x)=\{c(t): t \in T$ and $(s(t), e(t))=(S, x)\}$. Clearly, $C$ is a well-defined selection function.

Now consider the following procedure to move from a selection funtion to a data set. Suppose $X=\mathbb{R}^{m}, \mathcal{D}$ is finite, and $C: \mathcal{D} \rightarrow \mathcal{P}(X)=\mathcal{P}\left(\mathbb{R}^{m}\right)$ is a selection function. Let

$$
|T|=\sum_{(S, x) \in \mathcal{D}}|C(S, x)|
$$

and $T=\{1, \ldots,|T|\}$. Let $g:\{1, \ldots,|\mathcal{D}|\} \rightarrow \mathcal{D}$ be a bijection. For all $j \in\{1, \ldots,|\mathcal{D}|\}$, let $f_{j}:\{1, \ldots,|C(g(j))|\} \rightarrow C(g(j))$ be a bijection.

For all $t \in\{1, \ldots,|C(g(1))|\}$, let

$$
(s(t), e(t), c(t))=\left(g(1), f_{1}(t)\right)
$$

For all $k \in\{2, \ldots,|\mathcal{D}|\}$ and for all $t \in\left\{\sum_{j=1}^{k-1}|C(g(j))|+1, \ldots, \sum_{j=1}^{k-1}|C(g(j))|+|C(g(k))|\right\}$ (provided that $|\mathcal{D}|>1$ ), let

$$
(s(t), e(t), c(t))=\left(g(k), f_{k}\left(t-\sum_{j=1}^{k-1}|C(g(j))|\right)\right) .
$$

The above constructions allow us to go back and forth between selection functions and data sets. Note that, of course, the data set derived from a selection function is not unique because the assignment of triples of feasible sets, reference points, and chosen points to $T$ is arbitrary.

\section{References}

Arrow, K.J. (1959), "Rational choice functions and orderings," Economica 26, 121-127. Bossert, W. And Sprumont, Y. (2000), "Core rationalizability in two-agent exchange economies," Working Paper 2000-07, Department of Economics, Rice University. 
Camerer, C. (1994), "Individual decision making," in: Kagel, J. And Roth, A. (eds.), Handbook of Experimental Economics, Princeton University Press, Princeton, 587704 .

Houthakker, H.S. (1950), "Revealed preference and the utility function," Economica 17, 159-174.

Richter, M.K. (1966), "Revealed preference theory," Econometrica 34, 635-645.

Richter, M.K. (1971), "Rational choice," in: Chipman, J.S., Hurwicz, L., RichTer, M.K., And Sonnenschein, H.F. (eds.), Preferences, Utility, and Demand, Harcourt Brace Jovanovich, New York, 29-58.

Samuelson, P.A. (1938), "A note on the pure theory of consumer's behaviour," Economica 5, 61-71.

Samuelson, P.A. (1948), "Consumption theory in terms of revealed preference," Economica 15, 243-253.

SEn, A. (1971), "Choice functions and revealed preference," Review of Economic Studies 38, 307-317.

Shafir, E. AND Tversky, A. (1995), "Decision making," in: Osherson, D.N. AND Smith, E.E. (eds.), Invitation to Cognitive Science: Thinking, MIT Press, Cambridge, 77-109.

Simon, H.A. (1955), "A behavioral model of rational choice," Quarterly Journal of Economics 69, 99-118.

Simon, H.A. (1956), "Rational choice and the structure of the environment," Psychological Review 63, 129-138.

SzPilrajn, E. (1930), "Sur l'extension de l'ordre partiel," Fundamenta Mathematicae 16, 386-389.

Zhou, L. (1997), "Revealed preferences: the role of the status quo," mimeo, Department of Economics, Duke University. 